\title{
Spectral Lines as Light Sources in Polariscopic ${ }^{1}$ Measurements.
}

By Frederick Bates.

D ECENT progress in polariscopic work necessitates careful consideraR tion of the character and behavior of the light sources used. Unless the source can be accurately defined and its constancy depended upon it is obviously impossible to compare the polariscopic measurements of different observers.

Let two spectral lines with unequal intensities be considered as a source of light for a half shade polariscope. It is found from a theoretical development that a rotation is measured which differs from the rotation that would have been obtained, had the lines been of equal intensity, by an angle

$$
\delta= \pm \frac{1}{2} \frac{K-\mathrm{I}}{K+\mathrm{I}}\left(\alpha_{1}-\alpha_{2}\right)
$$

where $K$ is the ratio of the intensities and $\left(\alpha_{1}-\alpha_{2}\right)$ is a function of the rotation dispersion.

By means of (I) we can calculate the optical center of gravity of the sodium lines and the errors in measuring rotations with these lines as the source of light.

The use of the line 546. I $\mu$ obtained from incandescent mercury vapor is advocated as the international standard source for all polariscopic measurements. In order to make possible the use of this source where the rotation value for quartz is desired in terms of the sodium lines a determination was made of the temperature coefficient and also the ratio of the rotation for the proposed source and the sodium lines.

We have thus the formula

$$
\alpha_{t}=\alpha_{0}(\mathrm{I}+0.000 \mathrm{I} 44 t),
$$

giving the rotation for quartz at any temperature between $4^{\circ} \mathrm{C}$. and $50^{\circ}$ C. for the line 546. I $\mu$. To give the rotation in terms of the sodium lines we have

$$
\frac{\sigma_{\lambda}=546 . \mathrm{I}}{\sigma_{\lambda}=5^{89.25}}=\mathrm{I} . \mathrm{I} 75^{\mathrm{I} 66} \pm 0.0000 \mathrm{I} 2 .
$$

${ }^{1}$ Abstract of a paper presented at the meeting of the Physical Society held April 20-2I, I906. 